\section{Database Trigger}

Mikael Berndtsson and Jonas Mellin University of Skövde, Skövde, Sweden

\section{Synonyms}

Triggers

\section{Definition}

A database trigger is code that is executed in response to events that have been generated by database commands such as INSERT, DELETE, or UPDATE.

\section{Key Points}

Triggers are code that are executed in response to events that have been generated before or after a database operation. They are sometimes separated as pre- and post-triggers in the literature. A pre-trigger can be used as an extra validation check before the database command is executed, whereas a post-trigger can be used as a notification that the database command has been executed.
Triggers can be classified according to trigger granularity: row-level triggers or statement-level triggers. In case of row-level triggers, each row will generate an event, whereas statement-level triggers occur only once per database command.

Overviews of database triggers can be found in $[1,2]$.

\section{Cross-References}

- Active Database (aDB)

- Active Database (Management) System (aDBS/aDBMS)V

$\checkmark$ ECA Rules

$\checkmark$ Event

\section{Recommended Readings}

1. Kulkarni KG, Mattos NM, Cochrane R. Active database features in SQL3. In: Active rules in database systems; 1999. p. 197-219.

2. Sudarshan S, Silberschatz A, Korth H. Triggers, chap. 8.6; 2006. p. 329-34. 